Portland State University

PDXScholar

\title{
Perceived Experiences of Discrimination in Health Care: A Barrier for Cancer Screening Among American Indian Women with Type 2 Diabetes
}

Kelly Gonzales

Portland State University, kelly.gonzales@pdx.edu

Anna K. Harding

Portland State University

William E. Lambert

Portland State University

Rongwei Fu

Oregon Health \& Science University

William G. Henderson

University of Colorado

Follow this and additional works at: https://pdxscholar.library.pdx.edu/sph_facpub

Part of the Women's Health Commons

Let us know how access to this document benefits you.

\section{Citation Details}

Gonzales, Kelly; Harding, Anna K.; Lambert, William E.; Fu, Rongwei; and Henderson, William G., "Perceived Experiences of Discrimination in Health Care: A Barrier for Cancer Screening Among American Indian Women with Type 2 Diabetes" (2013). OHSU-PSU School of Public Health Faculty Publications and Presentations. 198.

https://pdxscholar.library.pdx.edu/sph_facpub/198

This Post-Print is brought to you for free and open access. It has been accepted for inclusion in OHSU-PSU School of Public Health Faculty Publications and Presentations by an authorized administrator of PDXScholar. Please contact us if we can make this document more accessible: pdxscholar@pdx.edu. 


\title{
Perceived Experiences of Discrimination in Health Care: A Barrier for Cancer Screening Among American Indian Women with Type 2 Diabetes
}

\author{
Kelly L. Gonzales, MPH, PhD ${ }^{a, b,}{ }^{*}$, Anna K. Harding, PhDa, William E. Lambert, $\mathrm{PhD}^{\mathrm{c}}$, \\ Rongwei Fu, $\mathrm{PhD}^{\mathrm{c}}$, and William G. Henderson, $\mathrm{MPH}, \mathrm{PhD}^{\mathrm{d}}$ \\ Kelly L. Gonzales: kelly.gonzales@pdx.edu \\ aSchool of Community Health, College of Urban \& Public Affairs, Portland State University, \\ Portland, Oregon \\ bSchool of Biological and Population Health Sciences, College of Public Health and Human \\ Sciences, Oregon State University, Corvallis, Oregon \\ 'Department of Public Health and Preventive Medicine, Oregon Health \& Science University, \\ Portland, Oregon \\ dUniversity of Colorado Health Outcomes Program, Aurora, Colorado
}

\section{Abstract}

Purpose-Breast and cervical cancer-mortality disparities are prominent among American Indian women. These disparities, in part, may result from patients perceived experiences of discrimination in health care. This report evaluates the impact of perceived discrimination on screening for breast and cervical cancer in a sample of 200 American Indian women with type 2 diabetes.

Methods-Data were collected from patient report and medical records. Prevalence of breast and cervical cancer screening were assessed. Unadjusted and adjusted logistic regression analyses were used to assess associations between perceived discrimination, cancer screening status, and patients' health care-seeking behaviors.

Findings-Substantial proportions of AI women in our sample were behind the recommended schedules of screening for breast and cervical cancer. Adjusted estimates revealed that perceived discrimination was significantly associated with not being current for clinical breast examination and Pap test, and was close to statistical significance with not being current for mammography. The number of suboptimal health care-seeking behaviors increased with higher mean levels of perceived discrimination.

Conclusions-Among AI women, perceived discrimination in health care may negatively influence use of breast and cancer screening services, and health care-seeking behaviors. More research is needed among AIs to examine features of health care systems related to the phenomenon patients perceived experience of discrimination. 


\section{Introduction}

American Indian (AI) women have higher mortality rates from breast and cervical cancer than non-Hispanic Whites (Espey, Paisano, \& Cobb, 2005; Northwest Portland Area Indian Health Board, 2011). One factor that helps to explain these disparities is that AI women are more likely than their non-Native counterparts to be diagnosed with cancer at later stages (Clegg, Hankey, Chu, \& Edwards, 2002). Among AI women, a late-stage cancer diagnosis strongly correlates with unmet cancer screening. AI women delay use of cancer screening services and generally receive fewer screenings than other groups (Eberth, Huber, \& Rene, 2010; Giuliano, Papenfuss, de Guernsey de Zapien, Tilousi, \& Nuvayestewa, 1998). Prominent barriers associated with cancer screening in AI women include availability of services and medical access (Coughlin, Leadbetter, Richards, \& Sabatino, 2002; Eberth et al., 2010; Schumacher, Slattery, \& Lanier et al., 2008); however, these factors do not entirely explain why AI women underuse cancer screening services.

Reducing cancer mortality rates among AI women may a require better understanding of patient experiences and their influence on patient engagement. For example, two recent studies demonstrate that, among AI women, a positive patient- provider relationship is associated with more favorable Pap test experiences and being current on breast and cervical cancer screening (Simonds, Christopher, Sequest, Colditz, \& Rudd, 2011; Smith, Chriostopher, Lafromboise, Letiecq BL, \& McCormick, 2008). However, little is known about the specific features of the patient experience for AI women that may undermine engagement in cancer screening.

A promising area of the research that links patient experiences and medical engagement is patients' perceived experiences of discrimination when obtaining health care (Bird \& Bogart, 2001; Call et al., 2006; Johansson, Jacobsen, \& Buchwald, 2006;Kressin, Raymond, \& Manze, 2008). Perceived discrimination in health care (hereinafter "perceived discrimination") is broadly defined as the belief that one has experienced unfair treatment in a medical setting based on characteristics including race, ethnicity, or other demographic or socioeconomic attributes (Kressin et al., 2008). Perceived discrimination has been associated with lower patient engagement, receipt of fewer health care services, and poorer health (Kressin et al., 2008; Shavers et al., 2012). The associations between perceived discrimination and cancer screenings, however, have been inconsistently demonstrated (Crawley, Ahn, \& Winkleby, 2008; Hausmann, Jeong, Bost, \& Ibrahim, 2008; Dailey, Kasl, Holford, \& Jones, 2007; Shariff-Marco, Klassen, \& Bowie, 2010; Simonds, Colditz, Rudd \& Sequist, 2011).

In this report, we use a multi-item measure to evaluate the impact of perceived discrimination on screening for breast and cervical cancer in a sample of AI women with type 2 diabetes, a group previously characterized to have low cancer screening despite routine access to health care (Giroux et al., 2000). We hypothesized that perceived discrimination is associated with cancer screening status among AI women. Specifically, we examined 1) status for breast and cervical cancer screening, 2) correlates associated with not being current on cancer screenings, 3 ) whether perceived discrimination is independently associated with cancer screening status, and 4) associations between perceived discrimination and health care-seeking behaviors.

\section{Methods}

During 2008, we collected survey and medical records data from a sample of $270 \mathrm{AI}$ women. The sample was generated from electronic patient medical records managed by four tribally administered health care facilities located on Indian reservations in the Northwest 
region of the United States. These facilities have similar systems for maintaining patient health data, medical staffing, and health-related services. Demographic information about providers was not obtained. Clinical breast examination and Pap testing were routinely available through the tribal facilities, but mammography was not.

Participant eligibility criteria were 18 years or older, confirmed diagnosis of diabetes at least 1 year preceding data collection, classified as an active patient, and living within the facility's service area. Recruitment of the sample involved a mailed invitation, follow-up postcards, and announcements made throughout the tribal community. Informed consent was obtained and a $\$ 10$ gift card was given to all participants.

The survey was developed by adopting items from published studies on perceived discrimination (Bird \& Bogart, 2001; Blanchard \& Lurie, 2004; Trivedi \& Ayanian, 2006). The survey instrument was pilot tested in one tribal community with a convenience sample of $20 \mathrm{AI}$ women who met the study criteria. We administered the participant survey by mail and at data collection events scheduled in the tribal communities. Of the 270 women identified as eligible, 215 (80\%) completed the survey. Fifteen surveys (7\%) were excluded from the analysis because of incompleteness, yielding a total of 200 for analysis. Fifty-seven percent of the surveys were completed by mail. Age, education, and duration of diabetes diagnosis were similar between patients who completed or did not complete surveys, and between patients who used different methods of survey completion.

The study protocol was approved by the tribal councils and committees that monitor research conducted within each participating community. Institutional review board approval was obtained from the Portland Area Office Indian Health Service, the National Indian Health Service, and Oregon State University.

\section{Study Outcomes}

Cancer screening services-The three outcome variables were self-reported cancer screening status for 1) clinical breast examination, 2) mammography, and 3) Pap test. Because the participants had multiple sources of health care, and checking all sources of medical records was beyond the scope of this project, we considered self-report to be the best source for determining cancer screening status. The 2008 Indian Health Service Standards of Care for Adults with Type 2 Diabetes include cancer screening guidelines and were used as benchmarks for cancer screening status (Indian Health Service [IHS], 2008). The guidelines recommend clinical breast examination beginning at age 20, followed with annual screening; mammogram every 1 to 2 years among women aged 40 to 49 , and annually among women 50 years of age or older; and Pap smear beginning within 3 years of onset of sexual activity or at age 21, with screening every 3 years at a minimum.

Status of clinical breast examination was measured by asking participants whether they had received a "breast examination from a doctor" within the previous year. Status of mammography screening and Pap testing was measured by asking participants if they had received these services, each using a five-item response (within the past year, more than 1 year ago and up to 3 years ago, more than 3 years ago and up to 5 years ago, more than 5 years ago, never had the service). We dichotomized mammography status as follows: Not current, as never had the service or had the service more than 3 years ago (for those aged 40-49) or had the service more than 1 year ago (for those aged $\geq 50$ years), or current otherwise. Main analysis for mammography was limited to patient's 40 years of age or older. We dichotomized Pap testing status as follows: Not current, as never had the service or had the service more than 3 years ago, and current otherwise. Main analyses for Pap testing excluded participants who reported that they have had a hysterectomy. All other participants satisfied the eligibility criteria for the cancer screenings. 


\section{Independent Variables}

Perceived discrimination-The main independent variable was perceived discrimination (Bird \& Bogart, 2001). Participants were asked, "Thinking about your experience getting health care, how often does each of the following happen to you because of your race (because you are Native/American Indian)?" Items were: 1) You are not treated with courtesy, 2) you are not treated with respect, 3) you receive poorer service than other people, 4) a doctor, nurse, or medical provider acts as if he or she thinks you are not smart, 5) A doctor, nurse, or medical provider acts as if he or she is afraid of you, 6) a doctor, nurse, or medical provider acts as if he or she is better than you, and 7) you feel like a doctor, nurse, or medical provider is not listening to what you are saying. Perceived discrimination was scored on a 5 -point Likert-type scale $(1=$ never, $2=$ rarely, $3=$ sometimes, $4=$ most of the time, and $5=$ always).

This multi-item measure, modified previously by Bird and Bogart from Williams' Everyday Discrimination Scale (Williams, Yan, Jackson, \& Anderson, 1997), has been used among African Americans and individuals with specific health conditions (Bird \& Bogart, 2001; Hausman, Kressin, Hanusa, \& Ibrahim, 2010; Bird, Bogart, \& Delahanty, 2004). This measure has not been previously used among AIs. Using the Kaiser criterion, a factor analysis of the multi-item measure of perceived discrimination among our sample revealed a one-factor solution accounting for $74 \%$ of the total variance of all seven-scale items. Factor loadings ranged from 0.77 to 0.90 . Good internal reliability was also evidenced (Cronbach's alpha, $a=0.94)$.

In the main analysis, we created a dichotomous version of the perceived discrimination variable, defined as follows: Any, reporting at least "rarely" to one or more of the scale items; and none, reporting "never" to all items (Bird \& Bogart, 2001; Hausmann et al., 2010). We used this dichotomous variable to assess the association between perceived discrimination and cancer screening in logistic regression analysis. We also calculated the mean perceived discrimination across the seven items and used the tertiles of the mean perceived discrimination to compare health care-seeking behaviors as described below.

Health care-seeking behaviors-Participants reporting any perceived discrimination answered seven additional yes/no questions about health care-seeking behaviors (Blanchard \& Lurie, 2004). Participants were asked, "How has this experience(s) [of discrimination] impacted how you seek health care?" Items were coded as 1 (put off or postponed health care), 2 (hesitant in getting health care), 3 (did not come back for a follow-up appointment), 4 (did not follow the doctor's advice), 5 (did not follow the treatment plan or get a needed test), 6 (avoided the respective health care provider involved with the discrimination), and 7 (no longer used the respective health care facility where the discrimination occurred; Blanchard \& Lurie, 2004).

Patient characteristics-Self-reported employment, education, and perceived health status were collected by the survey. Age, duration of diabetes diagnosis, and source of health insurance were collected from medical records data.

\section{Analysis}

Descriptive statistics were computed as percentages in each category of the variable. We used generalized estimating equations for logistic and linear regressions to account for clustering within each tribal community. We examined the bivariable logistic associations between each cancer screening outcome and participant characteristics, health care characteristics, and perceived discrimination. Covariates meeting the criterion for bivariable association $(p \leq .20)$ were candidate variables considered in multivariable models for 
perceived discrimination. A backward stepwise selection method was used to determine the final model. Variables identified as associated with perceived discrimination in previous research, regardless of their significance level, were included in the final model (Kressin et al., 2008). Linear regression was used to examine associations between perceived discrimination and health care-seeking behaviors. All analyses were completed using PASW Statistics 17 (IBM, Armonk, NY). An alpha level of 0.05 was considered for statistical significance.

\section{Results}

More of the participants were middle-aged (50-64 years of age), had attained a high school education or less, and were unemployed (Table 1). More had diabetes for 1 to 5 years, all had health insurance through the IHS, and $77 \%$ had additional sources of health insurance. Fifty-eight percent were not current for clinical breast examination, $45 \%$ were not current for mammography, and 39\% were not current for Pap testing. Sixty-seven percent perceived discrimination in their health care.

Results from bivariable association indicated that not being current on cancer screenings was associated with several participant characteristics (Table 2). Participants with health insurance coverage only through the IHS had a statistically significant increased odds for not being current on clinical breast examination (odds ratio [OR], 2.27; 95\% confidence interval lsqb;CIrsqb; 1.93-2.67), and a significantly increased odds for not being current on mammography (OR, 3.86; 95\% CI, 2.64-5.64), compared with those with additional insurance.

In unadjusted analysis, participants who perceived discrimination in their health care were more likely to not be current on clinical breast examination and mammography screening. A similar association was observed for Pap test, but failed to achieve statistical significance (Table 2). After adjusting for potential confounding variables, perceived discrimination was significantly associated with not being current for clinical breast examination and Pap test, and was close to statistical significance with not being current for mammography (Table 3).

Among the 133 participants who reported any perceived discrimination, the number of suboptimal health care-seeking behaviors increased with higher mean levels of perceived discrimination (Figure 1). The group with the highest perceived discrimination reported a mean of 4.7 suboptimal health care- seeking behaviors $(p<.001)$ compared with a mean of 2.2 suboptimal health care-seeking behaviors among those with the lowest amount of perceived discrimination. These relationships remained significant after adjusting for respondent characteristics.

\section{Discussion}

We found substantial proportions of AI women in our sample to be behind on the recommended schedules of screening for breast and cervical cancer, which is consistent with prior research among AIs (Northwest Portland Area Indian Health Board, 2011; Giuliano et al., 1998; Ebert et al., 2010; Simonds \& Colditz et al., 2011). Most of the women in our sample had multiple sources of health insurance that could be used to help them access cancer screening elsewhere. Our data show that IHS-only health insurance strongly correlated with unmet breast cancer screening, which is also consistent with prior research among AI women (Simonds \& Colditz et al., 2011). This is a reasonable finding for mammography, because these services are lacking within the Indian health care system where our sample was drawn. However, unlike mammography, clinical breast examination is readily available within this system, and IHS-only health insurance cannot sufficiently 
explain why the AI women in our sample were not current on clinical breast examination. Prior studies have linked source of health insurance coverage with unmet cancer screening among AI women, but the findings have been mixed (Schumacher et al., 2008; Risendal, Roe, DeZapein, Papenfuss, \& Giuliano, 1999; Giuliano et al., 1998; Simonds \& Colditz et al., 2011). Additionally, our analysis revealed significant associations between perceived discrimination and both cancer screenings and health care-seeking behaviors.

On unadjusted analyses, we observed significant associations between perceived discrimination and not being current on clinical breast examination and mammography. After adjusting for covariates the association between perceived discrimination and clinical breast examination held, but mammography was no longer significant and like the other cancer screening variables demonstrated an inverse relationship.

Similarly, Blanchard and Lurie (2004), Shariff-Marco and colleagues (2010), and Dailey and associates (2007) failed to demonstrate a significant association between perceived discrimination and mammography in multiracial groups. However, these studies may have limitations regarding the measurement used to assess perceived discrimination and cancer screening (Crawley et al., 2008). Alternatively, two recent studies found positive associations between perceived discrimination and not being up to date on mammography screening. Analyses of statewide data completed by Crawley and colleagues (2008) showed that women reporting perceived discrimination were less likely to have received a mammogram than those reporting no discrimination (OR, 0.52; 95\% CI, 0.51-0.54). Using this same dataset, analyses of 2,266 AI women by Simonds \& Colditz et al., 2011 demonstrated a similarly strong association between perceived discrimination and not being current on mammography screening (OR, $0.42 ; 95 \%$ CI, $0.20-0.89)$.

Our study is among the first to demonstrate an association between patients' perceived discrimination and not being current on Pap testing. Three prior studies among racially diverse samples failed to demonstrate a significant association between perceived discrimination and Pap testing (Blanchard \& Lurie, 2004; Hausmann et al., 2008; Simonds $\&$ Colditz et al., 2011). This discrepancy may be explained by our use of a multi-item measure to assess perceived discrimination, as compared with the single-item measures in previous reports, which may underestimate prevalence (Hausmann et al., 2008).

We also assessed the indirect association between perceived discrimination and patient engagement by examining patient reports of suboptimal health care-seeking behaviors. Among patients reporting perceived discrimination, we found mono-tonic associations between perceived discrimination and suboptimal health care-seeking behaviors, where an increase in the mean level of perceived discrimination was accompanied by an increase in the number of suboptimal health care-seeking behaviors reported. Hausmann and coworkers (2010), who used the same multi-item measure of perceived discrimination used in our study, also found that perceived discrimination was significantly associated with patient disengagement. However, studies that used a single-item measure of perceived discrimination report no associations between perceived discrimination and patient engagement (Crawley et al., 2008; Hausmann et al., 2008; Shariff-Marco et al., 2010; Simonds \& Colditz et al., 2011; Trivedi \& Ayanian, 2006). Our findings further support that a multi-item measure may be required to examine the influence of perceived discrimination on patient adherence and engagement (Hausmann et al., 2010).

The limitations of our study are as follows. First, our design is cross-sectional and precludes us from inferring causality between perceived discrimination and cancer screening. Second, our findings may not generalize across subgroups of AIs, particularly AI women without diabetes, those with health care outside the Northwest, and those who access different types 
of health care programs specific to AIs. Third, our study could not account for the potential influence of health care systems-level barriers and personal economic factors; these potential confounders are uncontrolled in our analysis. Fourth, our study considered a broad time frame, lifetime, to assess perceived discrimination, and a limited time frame, last three years, to assess cancer screening status. This incongruence may limit our ability to temporally link associations of perceived discrimination with cancer screening status, and contribute to our null finding between perceived discrimination and mammography. Although the same broad time frame, lifetime, has been used in prior studies that examine and report associations between discrimination and recent health care (Kressin et al., 2008; Shavers et al., 2012), this issue should be considered in future research. Finally, we cannot determine from our data whether the women perceived discrimination in care they receive within or outside the Indian health care system. Future research should more closely examine this issue, along with features of the health care system related to perceived discrimination.

Our design offers several strengths over previous investigations of these issues. We had a high response rate with confirmation of important eligibility criteria for participation. Although we used a self-reported measure of most recent cancer screening, our estimates of screening status are similar to those previously reported among Northwest AI women (Northwest Portland Area Indian Health Board, 2011). We used a robust measure for perceived discrimination that included seven different aspects of participants' experiences in receiving health care. Our statistical methods accounted for potential correlation of participants within facilities (tribal reservation communities). Our sample, composed of AI women with a chronic health condition, may represent a group with more opportunities for interacting with the health care system than the groups currently represented in the literature on perceived discrimination.

\section{Implications for Practices and/or Policy}

Our findings have important implications for future research and provider practice. The results of our study clearly indicate that more research is needed to confirm the correlation between perceived discrimination and cancer screening among AI women. They also suggest three recommendations for the methods and subjects of this research.

First, future research into the correlation between perceived discrimination and cancer screening among AI women must use standardized measures of perceived discrimination, preferably multi-item measures that are specific to health care settings, with acceptable psychometric properties (Hausmann et al., 2010; Kressin et al., 2008). These studies should use similar time frames for both perceived discrimination and cancer screening events (Kressin et al., 2008).

Second, the high proportion of perceived discrimination among AI women in our sample indicates the need to expand the scope of this research to examine features of health care systems related to the phenomenon of perceived discrimination. Examining features of health care systems that service AI women, including the historical context and care practices through which care is provided, may lead to better knowledge about mechanisms and processes through which discrimination operates. For example, as argued previously by native leaders and researchers, the recent history of sterilization of some AI women without their consent may have an important and underexamined influence on how AI women view their health care and engage with the system (Lawrence, 2000; Simoni, Sehgal, \& Walters, 2004). This history may help to explain our study's findings of a significant association between perceived discrimination and both Pap testing and clinical breast examination. Relatedly, our null finding between perceived discrimination and mammography may be owing to growing efforts to improve mammography screening rates among AI women through community-based approaches such as mobile mammography clinics, on-site 
mammography through health fairs and other community events, and the use of culturally relevant messaging. It is possible that such features of community-based approaches help to mediate the potentially harmful links between perceived discrimination and mammography among AI women.

Third, our findings suggest that future research into perceived discrimination and cancer screening among AI women should consider the influence of provider behavior on patient perceptions and engagement. Numerous studies have documented that discrimination operates at multiple levels of health care systems, including the patient-provider encounter, and may stem from unconscious bias and stereotyping among providers (Institute of Medicine, 2003). Researchers suggest that provider bias and its influence on the patientprovider encounter may become pronounced in under-resourced health care settings, particularly among non-minority health care providers with little experience interacting with under-represented populations (Burgess, Fu, \& Van Ryn, 2004).

Our findings strongly suggest the need for further research into the correlation between perceived discrimination and cancer screening among AI women, and we have outlined three directions for that research. However, even if further research is not pursued, the associations found between perceived discrimination and cancer screening use are strong enough to suggest the need for the implementation of culturally responsive care practices. The use of culturally responsive health care practices throughout systems that serve AI women may help to both dismantle provider bias and stereotyping, and to improve disease prevention and patient engagement (Guadagnolo et al., 2009; Wexler \& Gone, 2012).

"Culturally responsive" care embraces current cultural competency practice, but it also goes further in that it integrates patient-level health beliefs, expectations, and cultural practices (Ring, 2009). Culturally responsive care may be particularly important in systems with distinct cultural and racial differences between patients and providers, and providers with limited experience interacting with patients from under-represented racial and ethnic groups. Thus, the implementation of culturally responsive care practices may be particularly beneficial for AI women whether they receive their health care within or outside the Indian health care system.

Finally, our findings suggest that the importance of raising awareness about discrimination in health care should not be underestimated. Discrimination awareness should be inclusive of AI patients, tribal leadership, and health care providers and administrators, both within and outside the Indian health care system. These efforts will foster new knowledge about the source of discrimination and its influence on cancer screening among AI women, and may generate new interventions to reduce or eliminate discrimination in health care systems that serve this population.

\section{Acknowledgments}

This research was supported by a number of awards received by Dr. Gonzales including: National Institute of Diabetes and Digestive and Kidney Diseases Award Number F31DK082279; National Cancer Institute (NCI) grant no. UO1-CA 86098; Northwest Health Foundation (NWHF) grant no. F03890; and Northwest Native American Research Center for Health (NW NARCH) grant no. U26IHS300003. The content is solely the responsibility of the authors and does not necessarily represent the official views of the National Institute of Diabetes and Digestive and Kidney Diseases or the National Institutes of Health. There are no financial conflicts of interest involving any of the co-authors.

The author express deep gratitude to the AI women who shared their experiences, and to the Tribal Councils and the member tribes of the Northwest Portland Area Indian Health Board for approving this work. Dr. Gonzales who is independent of any funder or sponsor " had full access to all the data in the study and takes responsibility for the integrity of the data and the accuracy of the data analysis". 


\section{References}

Bird ST, Bogart LM. Perceived race-based and socioeconomic status (SES)-based discrimination in interactions with health care providers. Ethnicity and Disease. 2001; 11:554-563. [PubMed: 11572421]

Bird ST, Bogart LM, Delahanty DL. Health-related correlates of perceived discrimination in HIV care. AIDS Patient Care and STDS. 2004; 18:19-26. [PubMed: 15006191]

Blanchard J, Lurie N. R-E-S-P-E-C-T: Patient reports of disrespect in the health care setting and its impact on care. Journal of Family Practice. 2004; 53:721-730. [PubMed: 15353162]

Burgess DJ, Fu SS, Van Ryn M. Why do providers contribute to disparities and what can be done about it? Journal of General Internal Medicine. 2004; 19:1154-1159. [PubMed: 15566446]

Call KT, McAlpine DD, Johnson PJ, Beebe TJ, McRae JA, Song Y. Barriers to care among American Indians in public health care programs. Medical Care. 2006; 44:595-600. [PubMed: 16708009]

Clegg LX, Li FP, Hankey BF, Chu K, Edwards BK. Cancer survival among US whites and minorities: A SEER (Surveillance, Epidemiology, and End Results) Program population-based study. Archives of Internal Medicine. 2002; 162(17):1985-1993. [PubMed: 12230422]

Coughlin SS, Leadbetter S, Richards T, Sabatino SA. Contextual analysis of breast and cervical cancer screening and factors associated with health care access among United States women, 2002. Social Sciences and Medicine. 2008; 66:260-275.

Crawley LM, Ahn DK, Winkleby MA. Perceived medical discrimination and cancer screening behaviors of racial and ethnic minority adults. Cancer Epidemiology, Biomarkers \& Prevention. 2008; 17:1937-1944.

Dailey AB, Kasl SV, Holford TR, Jones BA. Perceived racial discrimination and nonadherence to screening mammography guidelines: Results from the race differences in the screening mammography process study. American Journal of Epidemiology. 2007; 165:1287-1295. [PubMed: 17351294]

Huber JM, Eberth JC Jr, Rene A. Breast cancer screening practices and correlates among American Indian and Alaska Native women in California, 2003. Women's Health Issues. 2010; 20:139-145. [PubMed: 20211430]

Espey D, Paisano R, Cobb N. Regional patterns and trends in cancer mortality among American Indians and Alaska Natives, 1990-2001. Cancer. 2005; 103:1045-1053. [PubMed: 15685622]

Giroux J, Welty TK, Oliver FK, Kaur JS, Leonardson G, Cobb N. Low national breast and cervical cancer-screening rates in American Indian and Alaska Native women with diabetes. Journal of the American Board of Family Practice. 2000; 13:239-245. [PubMed: 10933287]

Giuliano A, Papenfuss M, de Guernsey de Zapien J, Tilousi S, Nuvayestewa L. Breast cancer screening among southwest American Indian women living on-reservation. Preventive Medicine. 1998; 27:135-143. [PubMed: 9465364]

Guadagnolo BA, Cina K, Helbig P, Molloy K, Reiner M, Cook EF, et al. Medical mistrust and less satisfaction with health care among Native Americans presenting for cancer treatment. Journal of Health Care for the Poor and Underserved. 2009; 20:210-226. [PubMed: 19202258]

Hausmann LR, Jeong K, Bost JE, Ibrahim SA. Perceived discrimination in health care and use of preventive health services. Journal of General Internal Medicine. 2008; 23:1679-1684. [PubMed: 18649109]

Hausmann LR, Kressin NR, Hanusa BH, Ibrahim SA. Perceived racial discrimination in health care and its association with patients' healthcare experiences: Does the measure matter? Ethnicity \& Disease. 2010; 20:40-47. [PubMed: 20178181]

Indian Health Service [HIS]. IHS Standards of care for adults with type 2 diabetes. 2008. Available from http://www.ihs.gov/MedicalPrograms/Diabetes/index.cfm?module=SOCCancer

Smedley, BD.; Stith, AY.; Nelson, AR., editors. Institute of Medicine. Unequal treatment: Confronting racial and ethnic disparities in health care. Washington, DC: National Academies Press; 2003.

Johansson P, Jacobsen C, Buchwald D. Perceived discrimination in health care among American Indians/Alaska Natives. Ethnicity \& Disease. 2006; 16:766-771. [PubMed: 17061725] 
Kressin NR, Raymond KL, Manze M. Perceptions of race/ethnicity-based discrimination: A review of measures and evaluation of their usefulness for the health care setting. Journal of Health Care for the Poor and Underserved. 2008; 19:697-730. [PubMed: 18677066]

Lawrence J. The Indian Health Service and the sterilization of Native American women. The American Indian Quarterly. 2000; 24:400-419.

Northwest Portland Area Indian Health Board. Cancer among Northwest American Indians and Alaska Natives. Portland, OR: Northwest Tribal Epidemiology Center; 2011.

Ring J. Psychology and medical education: Collaborations for culturally responsive care. Journal of Clinical Psychology in Medical Settings. 2009; 16:120-126. [PubMed: 19153824]

Risendal B, Roe D, DeZapein J, Papenfuss M, Giuliano A. Influence of health care, cost and culture on breast cancer screening: Issues facing urban American Indian women. Preventive Medicine. 1999; 29:501-509. [PubMed: 10600431]

Schumacher M, Slattery M, Lanier A, Ma KN, Edwards S, Ferucci ED, et al. Prevalence and predictors of cancer screening among American Indian and Alaska Native people: The EARTH study. Cancer Causes and Control. 2008; 19:725-737. [PubMed: 18307048]

Shariff-Marco S, Klassen AC, Bowie JV. Racial/ethnic differences in self-reported racism and its association with cancer-related health behaviors. American Journal of Public Health. 2010; 100:364-374. [PubMed: 20019302]

Shavers VL, Fagan P, Jones D, Klein WM, Boyington J, Moten C, et al. The state of research on racial/ethnic discrimination in the receipt of health care. American Journal of Public Health. 2012; 102:953-966. [PubMed: 22494002]

Simonds VW, Christopher S, Sequist TD, Colditz GA, Rudd RE. Exploring patient-provider interactions in a Native American community. Journal of Health Care for the Poor and Underserved. 2011; 22:836-852. [PubMed: 21841282]

Simonds VW, Colditz GA, Rudd RE, Sequist TD. Cancer screening among Native Americans in California. Ethnicity \& Disease. 2011; 21:202-209. [PubMed: 21749025]

Smith AJ, Christopher S, Lafromboise VR, Letiecq BL, McCormick AK. Apsaalooke women's experiences with Pap test screening. Cancer Control. 2008; 15:166-173. [PubMed: 18376384]

Simoni JM, Sehgal S, Walters KL. Triangle of risk: Urban American Indian women's sexual trauma, injection drug use, and HIV sexual risk behaviors. AIDS and Behavior. 2004; 8:33-45. [PubMed: 15146132]

Trivedi AN, Ayanian JZ. Perceived discrimination and use of preventive health services. Journal of General Internal Medicine. 2006; 21:553-558. [PubMed: 16808735]

Wexler LM, Gone JP. Culturally responsive suicide prevention in indigenous communities: Unexamined assumptions and new possibilities. American Journal of Public Health. 2012; 5:800806. [PubMed: 22420786]

Williams DR, Yan Y, Jackson JS, Anderson NB. Racial differences in physical and mental health: Socio-economic status, stress and discrimination. Journal of Health Psychology. 1997; 2:335-351. [PubMed: 22013026]

\section{Biographies}

Kelly L. Gonzales, MPH, PhD, is an Assistant Professor of the School of Community Health in the College of Urban \& Public Affairs at PSU. Her research focuses on diabetes-related health disparities experienced by AI/ANs, emphasizing connections with discrimination and health care.

Anna K. Harding, PhD, is a Professor and Co-Director of the School of Biological \& Population Health Sciences in the College of Public Health \& Human Sciences at OSU. Her research expertise is in environmental contamination and population-based risk, tribal environmental exposures, and community engagement. 
Dr. William E. Lambert, PhD, is Associate Professor and Head of the Division of Epidemiology in the OHSU Department of Public Health and Preventive Medicine. His research focuses on health disparities and the prevention of chronic disease.

Rongwei $\mathrm{Fu}, \mathrm{PhD}$, is an associate professor of biostatistics at OHSU Department of Public Health and Preventive Medicine. She has expertise in meta-analysis, Bayesian statistics, categorical data analysis and longitudinal models, and are interested in Bayesian metaanalysis, mixture models and applied methodological research in health science.

William G. Henderson, MPH, PhD, is a Professor of Biostatistics at the University of Colorado Health Outcomes Program and the Department of Biostatistics and Informatics, School of Public Health, University of Colorado Denver. Dr. Henderson's areas of expertise and interests are clinical trials and health outcomes research. 


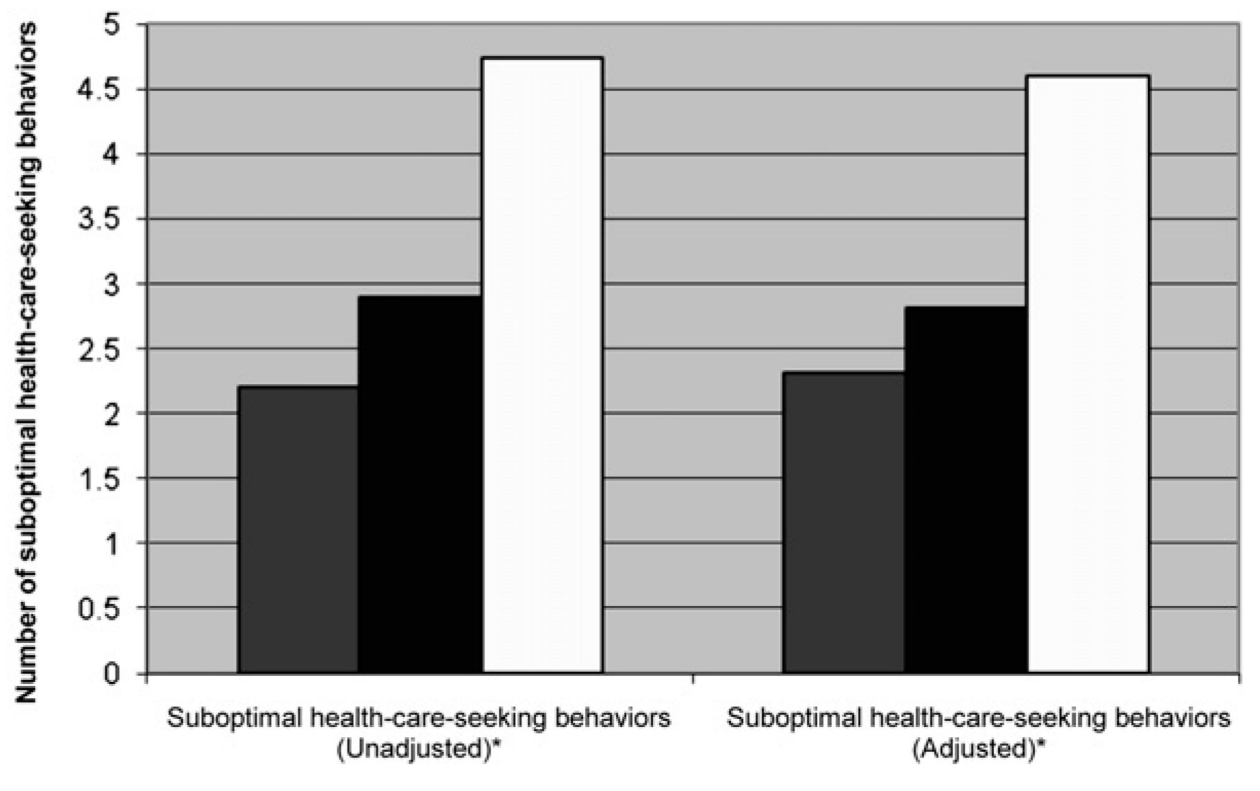

Terciles: Perceived discrimination

-Low (1.01-2.00) aModerate (2.01-2.86) aHigh (22.87)

Figure 1.

Suboptimal health care-seeking behaviors (out of a possible seven decisions) by mean perceived experiences of discrimination in health care terciles among American Indian

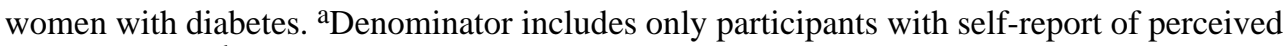
discrimination. ${ }^{b}$ Perceived discrimination tertiles: Low (1.01-2.00), moderate (2.01-2.86), and high ( $\geq 2.87$ ). ${ }^{\mathrm{c}}$ Adjusted for age, duration of diabetes, perceived health status, and source of health insurance. $* p \leq .001$. 
Table 1

Characteristics Among Sample of American Indian Women with Diabetes $(n=200)$

\begin{tabular}{|c|c|c|}
\hline Characteristic & Valid, $n(\%)$ & Missing, $n(\%)$ \\
\hline \multicolumn{3}{|l|}{ Demographic characteristics } \\
\hline Age (yrs) & & NA \\
\hline $18-49$ & $53(26)$ & \\
\hline $50-64$ & $87(44)$ & \\
\hline 265 & $60(30)$ & \\
\hline Education & & $3(2)$ \\
\hline Some high school & $32(16)$ & \\
\hline High school graduate/GED & $72(37)$ & \\
\hline Some college, vocational or trade school & $70(36)$ & \\
\hline College/professional school graduate & $23(12)$ & \\
\hline Employment Status & & $6(3)$ \\
\hline Full time & $56(29)$ & \\
\hline Part time & $17(9)$ & \\
\hline Unemployed & $69(36)$ & \\
\hline Retired & $52(27)$ & \\
\hline Duration of type 2 diabetes (yrs) & & NA \\
\hline $1-5$ & $89(45)$ & \\
\hline $6-10$ & $60(30)$ & \\
\hline$\geq 11$ & $51(26)$ & \\
\hline \multicolumn{3}{|l|}{ Perceived health status } \\
\hline Very good or better & $33(17)$ & NA \\
\hline Good & $86(43)$ & \\
\hline Poor or fair & $81(41)$ & \\
\hline Source of health insurance & & NA \\
\hline IHS only & $47(24)$ & \\
\hline IHS and other sources & $153(77)$ & \\
\hline \multicolumn{3}{|l|}{ Cancer screening } \\
\hline Clinical breast examination $(n=200)$ & & NA \\
\hline Not current & $116(58)$ & \\
\hline Mammography $(n=178)$ & & NA \\
\hline Not current & $80(45)$ & \\
\hline Pap test $(n=175)$ & & NA \\
\hline Not current & $68(39)$ & \\
\hline Perceived discrimination & & NA \\
\hline Any (rarely to at least one scale item) $(n=200)$ & $133(67)$ & \\
\hline
\end{tabular}

Abbreviations: GED, General Equivalency Diploma; HIS, Indian Health Service. 


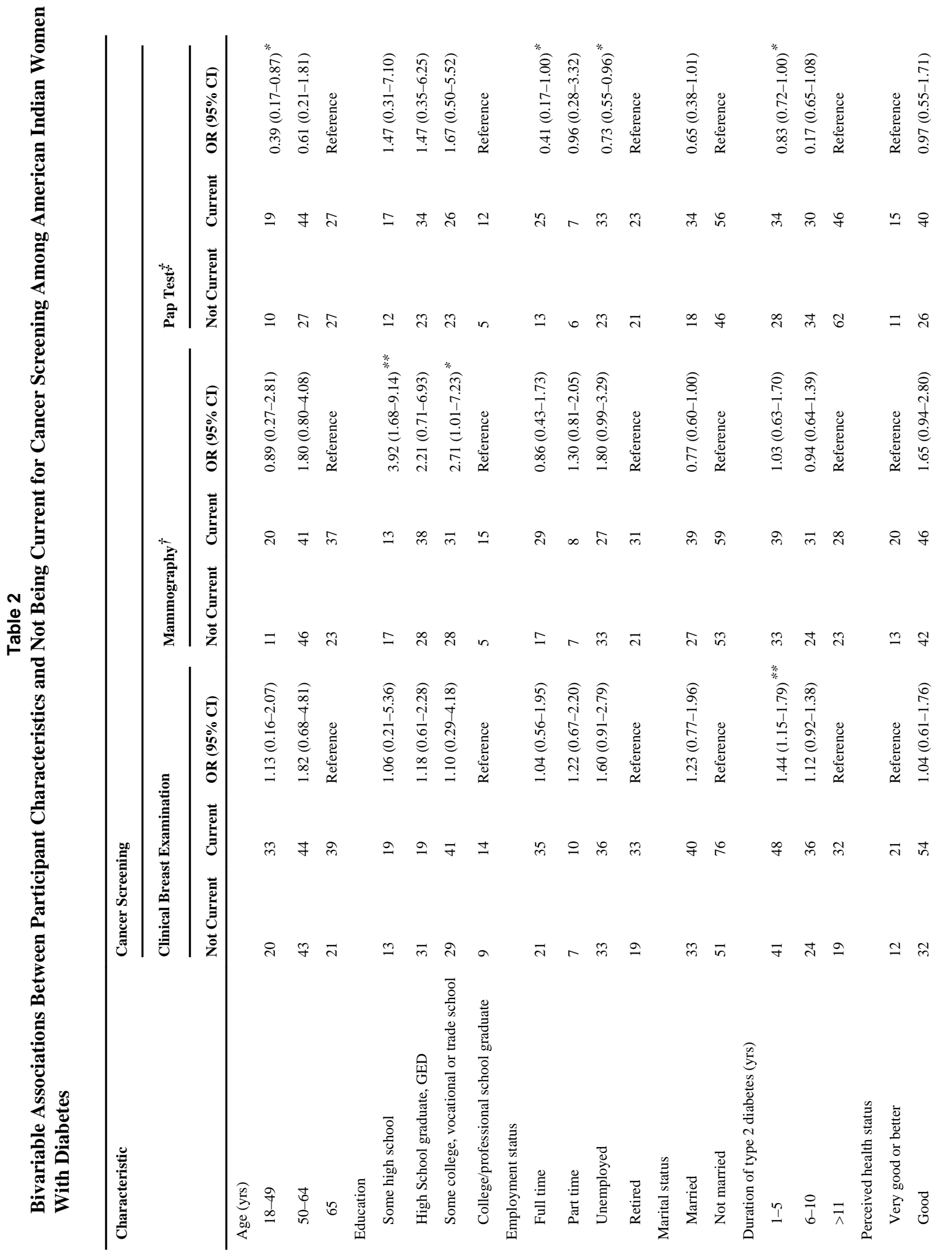




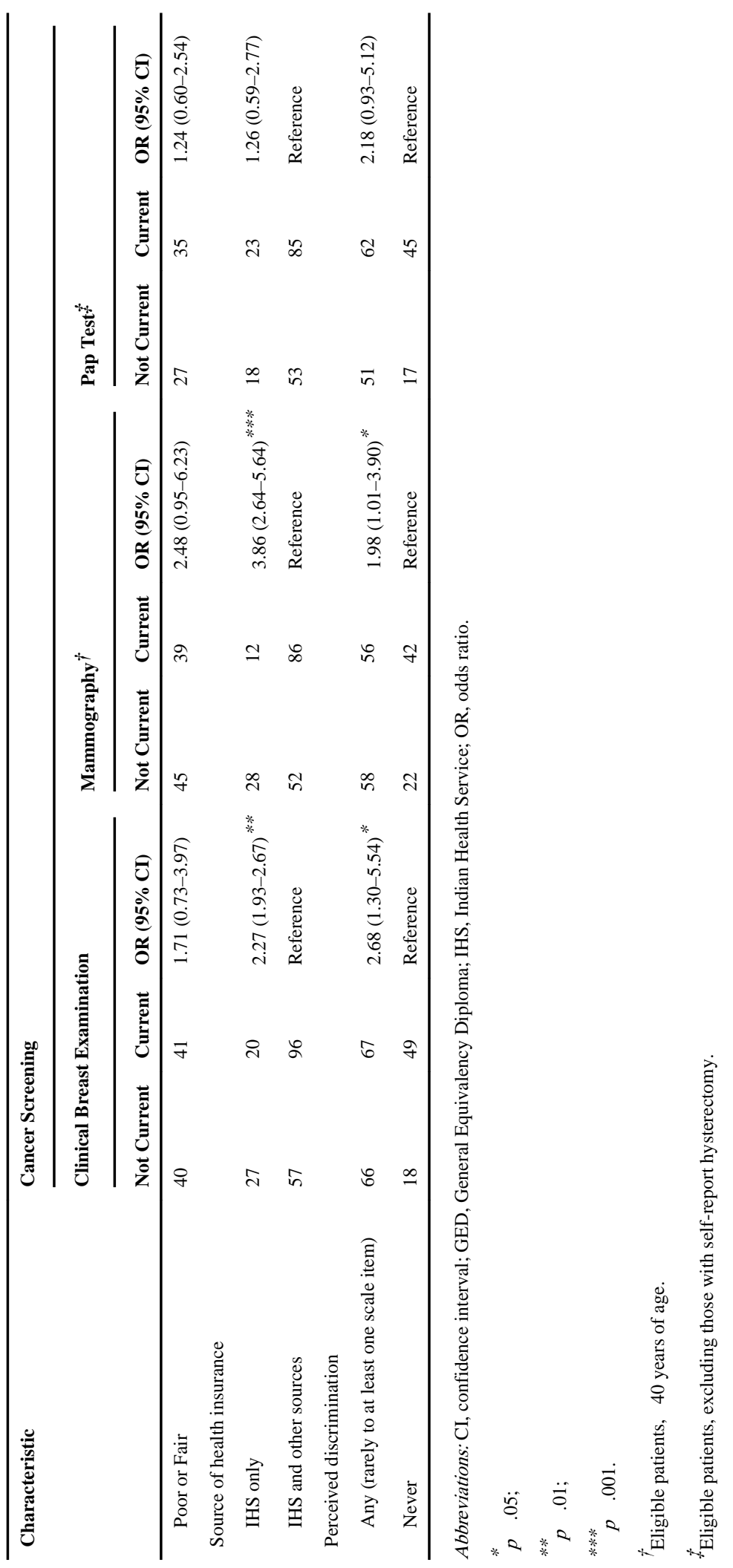


Table 3

Association Between Not Being Current on Cancer Screening and Perceived Experiences of Discrimination in Health Care in the Multivariable Model

\begin{tabular}{|c|c|c|c|}
\hline & \multicolumn{3}{|l|}{ Cancer-Related Screening } \\
\hline & Clinical Breast xamination ${ }^{\dagger}$ & Mammography $^{\dagger}$ & Pap test $^{\dagger}$ \\
\hline & $\operatorname{AOR}(95 \% \mathrm{CI})$ & $\operatorname{AOR}(95 \% \mathrm{CI})$ & $\operatorname{AOR}(95 \% \mathrm{CI})$ \\
\hline \multicolumn{4}{|l|}{ Perceived Discrimination } \\
\hline Any (rarely to at least one scale item) & $2.64(1.12-6.19)^{*}$ & $1.97(0.96-4.03)$ & $2.64(1.13-6.18)^{*}$ \\
\hline Never & Reference & Reference & Reference \\
\hline $\begin{array}{l}\text { Abbreviations: AOR, adjusted odds ratio; } \\
* \quad p<.05 \text {. }\end{array}$ & confidence interval. & & \\
\hline
\end{tabular}

